\title{
Dimensions of Impulsivity Are Associated with Poor Spatial Working Memory Performance in Monkeys
}

\author{
Alex S. James, ${ }^{1 \star}$ Stephanie M. Groman, ${ }^{1 \star}$ Emanuele Seu, ${ }^{1}$ Matthew Jorgensen, ${ }^{2}$ Lynn A. Fairbanks, ${ }^{2}$ and \\ J. David Jentsch ${ }^{1,2}$ \\ Departments of ${ }^{1}$ Psychology and ${ }^{2}$ Psychiatry and Biobehavioral Sciences, University of California, Los Angeles, Los Angeles, California 90095
}

\begin{abstract}
Impulsive behavior and novelty seeking are dimensions of temperament that are behavioral determinants of risk for attention deficit/ hyperactivity disorder and its neurocognitive endophenotypes, and variation in the dopamine $\mathrm{D}_{4}$ receptor gene (DRD4) explains at least a portion of the variance in the traits. To further characterize the dimensional phenotype associated with impulsiveness, adolescent male monkeys were evaluated using ecologically valid tests of impulsive approach and aggression in response to social or nonsocial stimuli; subsequently, a delayed response task was implemented to assess spatial working memory performance. Subjects were selected into this study based on their response to the social challenge task or by DRD4 genotype, resulting in three groups: low-impulsivity/common DRD4 allele, high-impulsivity/common DRD4 allele, or rare DRD4 allele. All animals acquired the delayed response task and could perform at near ceiling levels when a $\sim 0$ s delay version was imposed, but as delays were lengthened, high-impulsive animals, regardless of DRD4 genotype, made fewer correct responses than did low-impulsive subjects; an inverse relationship existed for working memory and impulsivity. Notably, impulsive behavior evoked by social and nonsocial stimuli explained overlapping and independent portions of the variance in working memory performance. CSF levels of monoamine metabolites did not significantly differentiate the high- and lowimpulsive animals, although monkeys carrying the DRD4 rare allele tended to exhibit higher monoamine turnover. These data indicate that dimensions of impulsivity may impact on working memory performance in qualitatively similar ways but through different mechanisms.
\end{abstract}

Key words: dopamine; ADHD; cognition; temperament; serotonin; prefrontal cortex

\section{Introduction}

Attention deficit/hyperactivity disorder (ADHD) is a multidimensional disorder that involves alterations in temperament (impulsivity), behavior (hyperactivity), and cognition (inattention, poor working memory) (Solanto, 2001; Castellanos and Tannock, 2002; Aron and Poldrack, 2005; Arnsten, 2006). Working memory (the ability to guide behavior based on representational knowledge) (Goldman-Rakic, 1987) is impaired in ADHD, and impulsivity and aspects of cognition, including working memory, are negatively related in people with ADHD (Chamberlain et al., 2007; Clark et al., 2007; Dowson et al., 2007). Furthermore, trait impulsiveness associates with differences in dopamine $\mathrm{D}_{2}$ receptor modulation of frontostriatal activation, assessed using functional magnetic resonance imaging, during working memory performance in a nonclinical sample (Cools et al., 2007), suggesting that the link between cognitive and temperamental phenotypes extends beyond the diagnosis of ADHD.

\footnotetext{
Received July 30, 2007; revised Nov. 14, 2007; accepted Nov. 14, 2007.

This work was supported by Public Health Service Grants P50-MH77248 and RL1-83270 (J.D.J.) and P40-RR19963 and R01-MH61852 (L.A.F.). We gratefully acknowledge the staff of the University of California, Los AngelesVeterans Affairs Vervet Research Colony for their participation in these studies and Sherry Breidenthal for designing and conducting the DRD4 genotyping.

*A.S.J. and S.M.G. contributed equally to this work.

Correspondence should be addressed to Dr. J. David Jentsch, Department of Psychology, University of California, Los Angeles, P.0. Box 951563, Los Angeles, CA 90095-1563. E-mail: jentsch@psych.ucla.edu.

DOI:10.1523/JNEUROSCI.4508-07.2007

Copyright $\odot 2007$ Society for Neuroscience $\quad$ 0270-6474/07/2714358-07\$15.00/0
}

Similar phenotypic associations might also be expected in animal models, permitting mechanistic studies that may illuminate the molecular and cellular bases of the linked phenotypes. Current animal models of ADHD capture some of the symptomatic features of the disorder (Davids et al., 2003; Russell et al., 2005; Sagvolden et al., 2005; Blondeau and Dellu-Hagedorn, 2007), but it remains to be shown that any rodent model exhibits multiple ADHD-related phenotypes (Dellu-Hagedorn, 2006; Blondeau and Dellu-Hagedorn, 2007).

Socially housed nonhuman primates exhibit naturally occurring variation in behavioral responses indicative of impulsivity (Higley et al., 1996; Fairbanks, 2001; Westergaard et al., 2003; Fairbanks et al., 2004), and in vervet monkeys, variation in impulsiveness is determined by significant genetic effects (Fairbanks et al., 2004). The genetic mechanisms may relate to the modulation of monoamine neurotransmitter function because impulsivity-related phenotypes inversely correlate with CSF levels of monoamine catabolites (Higley et al., 1996; Westergaard et al., 1999, 2003; Fairbanks et al., 2001).

One facet of impulsivity, novelty seeking, is associated with a 48 bp tandem-repeat polymorphism in exon 3 of dopamine $\mathrm{D}_{4}$ receptor gene (DRD4) in vervet monkeys (Bailey et al., 2007) that is similar in structure to a polymorphism found in the human DRD4 (Lichter et al., 1993) and that associates with the same phenotype in humans (Paterson et al., 1999; Lusher et al., 2001; Keltikangas-Jarvinen et al., 2003). Replicated associations be- 
tween DRD4 and ADHD (Smalley et al., 1998; Swanson et al., 2000; Faraone et al., 2005) may indicate that the link between DRD4 and novelty seeking penetrates to a syndromatic level and suggests that genetic variation in DRD4 may modulate ADHD endophenotypes.

Based on the mechanistic link between impulsive traits and the neural activation associated with working memory performance in humans (Cools et al., 2007), we reasoned that working memory performance in monkeys would be predicted by naturally occurring variation in responses evoked by ecologically valid measures of impulsivity. We hypothesized that greater impulsivity would be associated with poor working memory performance. Because variation in the DRD4 gene predicts a small portion of the variance in impulsive traits, we further examined this relationship in impulsive animals selected to carry common or rare DRD4 alleles. By contrasting monkeys carrying the impulsivitypromoting DRD4 allele with impulsive and nonimpulsive monkeys carrying the common DRD4 allele, we tested whether the as-of-yet unknown impulsivity-associated genetic mechanisms are linked to a signature set of phenotypes that are the same or different from that those linked to the DRD4 mechanism.

\section{Materials and Methods}

Subjects. The subjects in this study included 18 adolescent male vervet monkeys (Chlorocebus aethiops sabaeus) housed at the Association for Assessment and Accreditation of Laboratory Animal Care-accredited University of California, Los Angeles (UCLA)-Veterans Affairs Vervet Research Colony. The monkeys were housed in two social groups of nine members each; the two groups were composed of an equal mix of lowand high-impulsive monkeys and rare DRD4 allele carriers. The subjects were culled from their natal groups to form all-male groups at 42-52 months of age. The subjects were housed in outdoor enclosures equipped with an adjacent night room, containing a stainless-steel capture tunnel. Movement between the outdoor area and capture tunnel/night room was controlled using operable guillotine doors. Subjects were free to move about within the outdoor space or night room when testing was not being conducted.

Excess amounts of food were delivered each day, immediately after the completion of behavioral testing; water was always freely available in the outdoor area. No reduction in food availability was undertaken to facilitate behavioral testing. All methods for the care and use of nonhuman primates conformed to U.S. Department of Agriculture and Public Health Service standards, and all experimental protocols were approved by the Chancellor's Animal Research Committee at UCLA and the Institutional Animal Care and Use Committee for the Department of Veterans Affairs Greater Los Angeles Healthcare System.

Blood and CSF collection. Blood samples were collected from all Vervet Research Colony animals before initiation of this study, and genomic DNA was extracted for typing of the DRD4 tandem-repeat polymorphism. Genotyping was conducted, as previously described (Bailey et al., 2007). Approximately $10 \%$ of the colony members are heterozygous for the five-repeat variant of the exon 3 polymorphism (referred to, here, as DRD4.5), associated with increased novelty seeking; the remaining subjects were homozygous for the six-repeat allele (referred to as DRD4.6). The entire 2002 birth cohort was genotyped for the DRD4 allele before the initiation of these studies; six males were heterozygous for DRD4.5, and all were included as subjects in this study.

Between 49 and 59 months of age (during the period of cognitive testing), a $1 \mathrm{ml}$ sample of CSF was collected by cisternal puncture. This sampling was followed by a 2 week period off cognitive testing to avoid any influences of the anesthesia event on performance of the task. Anesthesia and analgesia was induced before sample collection by administration of ketamine $(8-10 \mathrm{mg} / \mathrm{kg}$, i.m.). Because high doses of ketamine are known to alter dopamine release and turnover in monkeys (Adams et al., 2002), all samples were collected within $15 \mathrm{~min}$ of anesthetic induction to minimize the impact of the anesthetic on monoamine metabolite levels in CSF. The samples were captured into microcentrifuge tubes, cooled on ice, and spun on a refrigerating centrifuge at $3000 \mathrm{rpm}$ for $15 \mathrm{~min}$; the top layer was aliquoted and subsequently frozen at $-80^{\circ} \mathrm{C}$ until the time of assay. Because of sampling problems, CSF was not collected from one subject.

Behavioral impulsivity measures. Behavioral impulsivity was measured in two ecologically valid challenges, the Intruder Challenge Test (Fairbanks, 2001; Fairbanks et al., 2001, 2004) and the Home Group Novelty Test (Bailey et al., 2007). Ecological validity is implied from studying the reaction of subjects within a context that bears some relationship to the natural ecological niche of the species (e.g., the response of a stable social group of monkeys to a stranger or to a novel inanimate stimulus at the edge of the home territory). Each subject was tested twice in the Intruder Challenge Test, once as a juvenile (28-38 months of age) and again as an adolescent (40-50 months of age), while still living in the natal group; the two scores were averaged to produce the values used in the current analyses. The Home Group Novelty Test was administered to these subjects when they were between 34 and 44 months of age.

The Intruder Challenge Test was used to measure social impulsivity and aggressiveness in each subject, while they were still living in their natal groups. During the test, all group members were confined to the night room, with the exception of three to four subjects who were in the outdoor portion. Each subject was observed continuously for $30 \mathrm{~min}$ after the placement of an unfamiliar adult animal (the "intruder") in an individual cage at the periphery of the enclosure boundary. Each subject was coded for latency to approach and for the occurrence of behaviors reflecting interest (approach within $1 \mathrm{~m}$, sit within $1 \mathrm{~m}$, sniff intruder, touch intruder), aggression (display to intruder, threaten intruder), redirected aggression, agitation (locomotion, vocalization), and anxiety (scratching, self-directed behavior), within each 1 min interval. Observers were trained and tested for reliability in behavior coding at regular intervals; $>90 \%$ interrater reliability was achieved with this scoring system. The Social Impulsivity Index was calculated as follows: [(30 - latency to approach the intruder) + the number of 1 min intervals within $1 \mathrm{~m}+$ sitting near + touching + sniffing + threatening + displaying to the intruder]. Previous research with a larger sample has shown that these behaviors fall into a single cohesive factor (Cronbach's $\alpha=0.84$ ) that combines rapidity of response with risky, assertive, and aggressive behaviors directed toward the intruder (Fairbanks, 2001). Animals scoring high on Social Impulsivity rush over to the unfamiliar adult male intruder immediately without taking the time to assess the situation. They place themselves at risk of injury by standing and sitting within arm's reach, and by touching, challenging, and threatening the stranger.

The Home Group Novelty Test was used to index novelty seeking, precisely as described previously (Bailey et al., 2007), except that different stimuli were used. During this test, a large, potentially threatening object (in this case, a 4-foot-tall artificial Christmas tree decorated with reflective streamers) was placed inside the home enclosure. A team of observers identified each individual that came within $1 \mathrm{~m}$ of the object in each 1 min interval for a $30 \mathrm{~min}$ test period. Latencies to approach shorter than $1 \mathrm{~min}$ were recorded to the nearest $10 \mathrm{~s}$. The object was selected to elicit a high enough level of interest and arousal to stimulate eventual approach in almost all of the subject population. The novelty seeking score was calculated as follows: [( $30-$ latency to approach in minutes $)+$ time spent in minutes within $1 \mathrm{~m}$ of the object], resulting in a potential range of $0-60$ for the measure.

Group assignment. Eighteen adolescent male subjects were selected into this study from the 2002 birth cohort that included a total of 26 males. Twenty of the 26 males carried the common DRD4.6 allele; of these, six animals scoring at or below the mean on the Social Impulsivity Index were selected for the low-impulsivity/DRD4.6 group and six scoring above the mean were selected for the high-impulsivity/DRD4.6 group. Six males from the 2002 birth cohort were heterozygous for the rare DRD4.5 allele, which is a mechanism known to modulate impulsivity (Bailey et al. 2007); all six were included in the third group in the experimental design, regardless of Social Impulsivity scores (DRD4.5).

Working memory performance: spatial delayed response task. At $\sim 4$ years of age, the subjects were trained to perform a three-choice spatial delayed response task in a custom-designed Wisconsin General Test apparatus that fit directly onto the capture tunnel within the night room of 
their enclosure. One at a time, monkeys were moved into the capture tunnel, and initially, all subjects were simply trained to reach through an aperture to open one of three identical boxes to obtain food rewards (e.g., banana slices, pieces of apple or grape).

Once the subjects were reliably opening all three boxes to obtain rewards, delayed response training was initiated. For the first stage, subjects watched while one of the three boxes was baited; the human tester visually confirmed that the monkey watched the box being baited at this, and all additional, stages of testing. After baiting, the screen was lowered and then immediately raised again ( $\sim 0 \mathrm{~s}$ "delay" between observing the box being baited and making a choice was imposed). When the screen was raised, the subject was allowed to open only one box; if he chose the rewarded box, he was allowed to keep the reward, the screen was lowered and a "correct choice" was scored. If he chose an unbaited box, the screen was immediately lowered, and an "incorrect choice" was scored. An omission was scored if the subject made no response within $30 \mathrm{~s}$. Subjects were tested three to five times per week, in sessions of 30 trials.

Subjects were trained until they performed at $80 \%$ accuracy in at least two consecutive training sessions (chance performance in this threechoice task is $33 \%$ ), at which point the delay was increased to $5 \mathrm{~s}$. A subset of monkeys achieved at least $80 \%$ correct responding in two consecutive sessions at $5 \mathrm{~s}$ and was moved to a $10 \mathrm{~s}$ delay; none of these monkeys were able to achieve $>80 \%$ choice accuracy at this longest delay in the period of training. A subset was unable to achieve $>50 \%$ accuracy at $5 \mathrm{~s}$, and for these subjects, the delay was reduced to $2.5 \mathrm{~s}$. The remaining monkeys achieved stable performance of $>50$ and $<80 \%$ accuracy at the $5 \mathrm{~s}$ delay; stable performance was determined when the monkeys achieved accuracy scores that varied by $<10 \%$ on 2 consecutive days. The rationale for this approach was to determine stable performance measures during a fixed delay condition that could reveal group effects as delays were increased. After reaching one of the above criteria, the monkeys were moved on to a variable delay task (below). Across this pretraining condition, the sessions to criteria at the $\sim 0 \mathrm{~s}$ delay, the total number of training sessions, the final delay achieved, and the accuracy at that final delay were our dependent measures.

After this initial period, the subjects were next exposed to a variable delay testing condition. For all subjects, one-third of their trials involved a $\sim 0$ s delay, one-third of their trials involved the final delay achieved during training $(2.5,5$, or $10 \mathrm{~s})$, and one-third of their trials involved a delay that was twice as long as their final training delay $(5,10$, or $20 \mathrm{~s}$, respectively). The subjects were exposed to two sessions of 30 trials each across 2 consecutive days; performance did not systematically vary across the $2 \mathrm{~d}$ (no main effect of day or day by group interaction), so the two sessions of data were pooled.

Monoamine metabolite analysis. Aliquots $(50 \mu \mathrm{l})$ of CSF were thawed on ice and injected directly on-column for analysis. The analytic system consisted of an isocratic, dual-piston pump (Shimadzu, Tokyo, Japan; model 10-ADVP), a reversed-phase column (Supelco, Bellefonte, PA; model SUPELCOSIL LC-18 $3 \mu \mathrm{m}, 15 \mathrm{~cm} \times 4.6 \mathrm{~mm}$ ), and an electrochemical detector system (Bioanalytical Systems, West Lafayette, IN; model Epsilon e5) equipped with a glassy-carbon, radial flow cell. The mobile phase was a $6 \%$ acetonitrile solution, containing $0.6 \%$ tetrahydrofuran, $0.1 \%$ diethylamine, $0.025 \mathrm{~mm}$ EDTA, $30 \mathrm{~mm}$ sodium citrate, $13.7 \mathrm{~mm}$ sodium phosphate, and $2.3 \mathrm{~mm}$ 1-octane-sulfonic acid, $\mathrm{pH} 2.95$. The applied potential was $+0.7 \mathrm{~V}$; the data were integrated on-line, referenced to external standards, and expressed as nanograms of analyte per $50 \mu \mathrm{l}$ of CSF.

Statistical analyses. Main effects and interactions were examined with factorial or repeated-measures ANOVA and were followed up by unpaired $t$ tests, where appropriate. Rank data (last delay achieved) was analyzed by Kruskal-Wallis test, and all correlations were estimated using simple or multiple regression analyses.

\section{Results}

\section{Behavioral impulsivity measures}

The mean (+SEM) behavioral impulsivity scores for the three experimental groups are shown in Figure 1. With respect to the Social Impulsivity scores, overall ANOVA detected a main effect of group $\left(F_{(2,15)}=11.0 ; p<0.001\right)$. The high- and low-
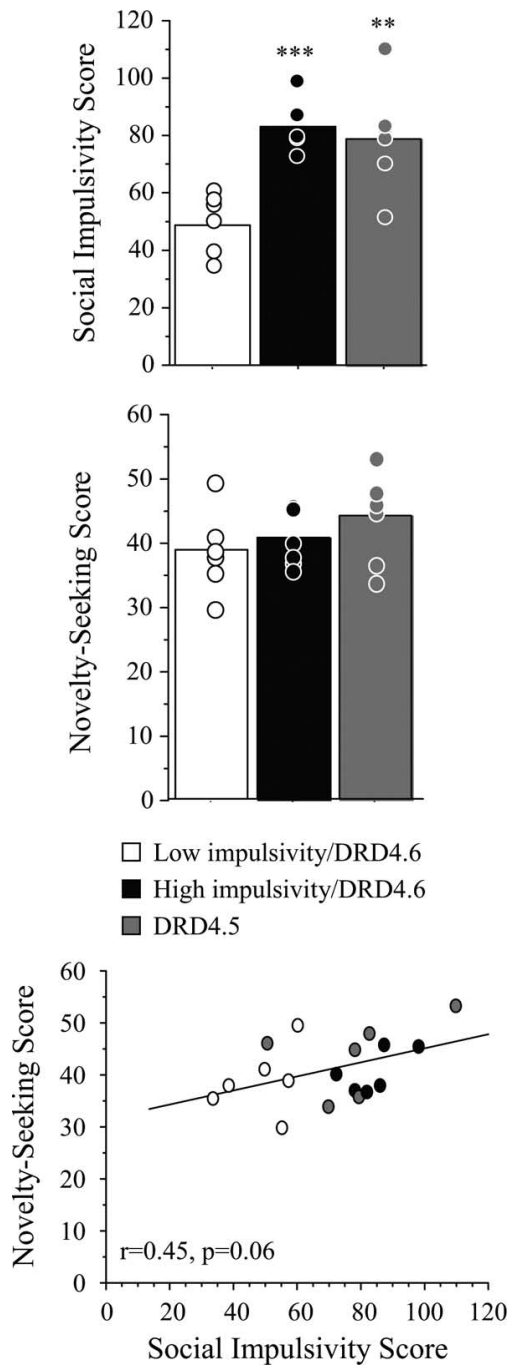

Figure 1. Social Impulsivity (top panel) and Novelty Seeking (middle panel) scores among the three groups of subjects included in this study. Both the high-impulsivity/DRD4.6 and DRD4.5 groups exhibited higher social impulsivity than the low-impulsivity/DRD4.6 group. The groups did not differ significantly according to the Novelty Seeking score. Social Impulsivity and Novelty Seeking scores were moderately correlated (bottom panel). Bars represent mean group data. Dots represent individual animal data points. ${ }^{* *} p<0.01$ and ${ }^{* * *} p<0.001$ : significantly different from the low-impulsivity/DRD4.6 group.

impulsivity groups with the common DRD4 allele were different by design (low-impulsivity group: mean \pm SEM, $48.9 \pm 4.4$; minimum, 33.5; maximum, 60; high-impulsivity group: mean \pm SEM, $83.3 \pm 3.7$; minimum, 72 ; maximum, $98 ; t_{(10)}=-6.0 ; p<$ $0.001)$. The six monkeys selected into the study because of DRD4.5 genotype scored higher than the low-impulsivity group and did not differ significantly from the high-impulsivity group on the Social Impulsivity Index (Fig. 1) (DRD4.5: mean \pm SEM, $78.6 \pm 7.9$; minimum, 50.5 ; maximum, $109.5 ; t_{(10)}=-3.3 ; p=$ $0.008)$. Although the Novelty Seeking score was not significantly different between the low-impulsivity/DRD4.6 group and either of the other two groups (Fig. 1) $\left(F_{(2,15)}=1.1 ; p=0.37\right)$, there was a strong trend for the Social Impulsivity score to correlate positively with the Novelty Seeking score in this small cohort (Fig. 1) (Pearson's $r=0.45 ; p=0.06$ ).

\section{Spatial delayed-response training}

All three groups acquired the rules of the spatial delayed-response task with equal capability. When trained on an initial $\sim 0$ s con- 
Table 1. Acquisition of the spatial delayed-response task

\begin{tabular}{|c|c|c|c|c|c|}
\hline Group & Total sessions at the 0 s delay & Final accuracy achieved at $0 \mathrm{~s}(\%)$ & Total no. of training sessions & Final delay achieved (s) & Final accuracy ( 5 s delays) (\%) \\
\hline Low impulsivity/DRD4.6 & $4.2 \pm 0.7$ & $90.8 \pm 0.9$ & $14.7 \pm 0.7$ & $5.0 \pm 0.0$ & $74.1 \pm 3.5$ \\
\hline High impulsivity/DRD4.6 & $6.2 \pm 1.2$ & $93.3 \pm 1.9$ & $15.0 \pm 0.8$ & $5.4 \pm 1.0$ & $59.2 \pm 5.8^{*}$ \\
\hline DRD4.5 & $4.7 \pm 0.6$ & $89.0 \pm 2.1$ & $14.7 \pm 0.8$ & $5.0 \pm 1.1$ & $77.8 \pm 4.7$ \\
\hline
\end{tabular}

Groups did not differ in the number of sessions required to learn a no-delay $(0 \mathrm{~s})$ version of the task or how accurately they performed under that condition. They also did not differ in the total number of training sessions delivered or the final delay achieved. However, high-impulsivity/DRD4.6 monkeys performed more poorly in response to a 5 s delay than did the low-impulsivity/DRD4.6 group ( $\left.{ }^{*} p<0.05\right)$. Data represent mean \pm SEM ( $n=6 /$ group).

Table 2. Final delays achieved as a function of group

\begin{tabular}{llll}
\hline & Low impulsivity/DRD4.6 & High impulsivity/DRD4.6 & DRD4.5 \\
\hline 2.5 s delay & 0 & 1 & 2 \\
5 s delay & 6 & 4 & 3 \\
10 s delay & 0 & 1 & 1 \\
\hline
\end{tabular}

Data represent the number of monkeys per group achieving the respective delay conditions. No group differences were found.

dition, there were no differences among the groups in the average number of sessions required to reach criterion performance ( $>80 \%$ accuracy in two consecutive daily sessions; chance accuracy in this task, $33 \%)$ (Table 1$)\left(F_{(2,15)}=1.5 ; p=0.27\right)$, nor in the final accuracy level obtained $\left(F_{(2,15)}=1.6,0.24\right)$. These data suggest that all monkeys were equally able to learn and perform the task when there was little or no mnemonic component.

The monkeys were then tested in additional sessions in which delays were individually titrated between $2.5,5$, and 10 s to determine a delay where each subject stably performed at $>50$ and $<80 \%$ accuracy. Table 1 shows that the total number of training sessions did not vary between the groups $\left(F_{(2,15)}=0.06 ; p=0.9\right)$, nor did the final delay reached after the individualized training regimen (Table 1) (corrected $H=0.43 ; p=0.8$ by KruskalWallis test). Most monkeys stabilized at a 5 s delay (Table 2 displays the distribution of final delays achieved in the three groups), and simple main effects analyses revealed that highimpulsivity/DRD4.6 monkeys performed poorer than low-impulsivity/DRD4.6 monkeys at this delay (Table 1$)(t=2.2 ; \mathrm{df}=$ $10 ; p=0.05)$, whereas DRD4.5 monkeys did not differ from the low-impulsivity/DRD4.6 group (Table 1$)(t=-0.63$; df $=10$; $p=0.5)$. The presence of performance impairments at $5 \mathrm{~s}$, but not $0 \mathrm{~s}$, suggest a specific retention deficit in the high-impulsivity/ DRD4.6 monkeys.

\section{Variable delay testing}

Subsequently, variable delay versions of the task that increased the retention requirements were imposed (one-third of the trials involved a delay that was twice as long as the final training delay achieved), and group differences were apparent (Fig. 2). Data from two variable delay sessions were pooled and subjected to ANOVA. Overall, there were main effects of group $\left(F_{(2,15)}=4.3\right.$; $p=0.03)$ and delay $\left(F_{(2,30)}=90.7 ; p<0.0001\right)$, as well as a group by delay interaction $\left(F_{(4,30)}=3.0 ; p=0.03\right)$. Post hoc comparisons revealed that none of the groups differed from one another under the $\sim 0$ s delay condition; however, the high-impulsivity/ DRD4.6 group performed more poorly than the low-impulsivity/ DRD4.6 at the middle $\left(t_{(10)}=2.8 ; p=0.02\right)$ and long $\left(t_{(10)}=2.0\right.$; $p=0.07)$ delays, whereas the DRD4.5 group differed from the low-impulsivity/DRD4.6 at only the longest delays $\left(t_{(10)}=2.8\right.$; $p=0.02$ ). None of the groups differed in the number of omissions, which virtually never occurred in this cohort.

Response accuracy when the long delays were imposed was regressed against the Social Impulsivity score to test whether the relationship between these two variables held at an individual level. Considering all 18 subjects, the relationship was highly sig-
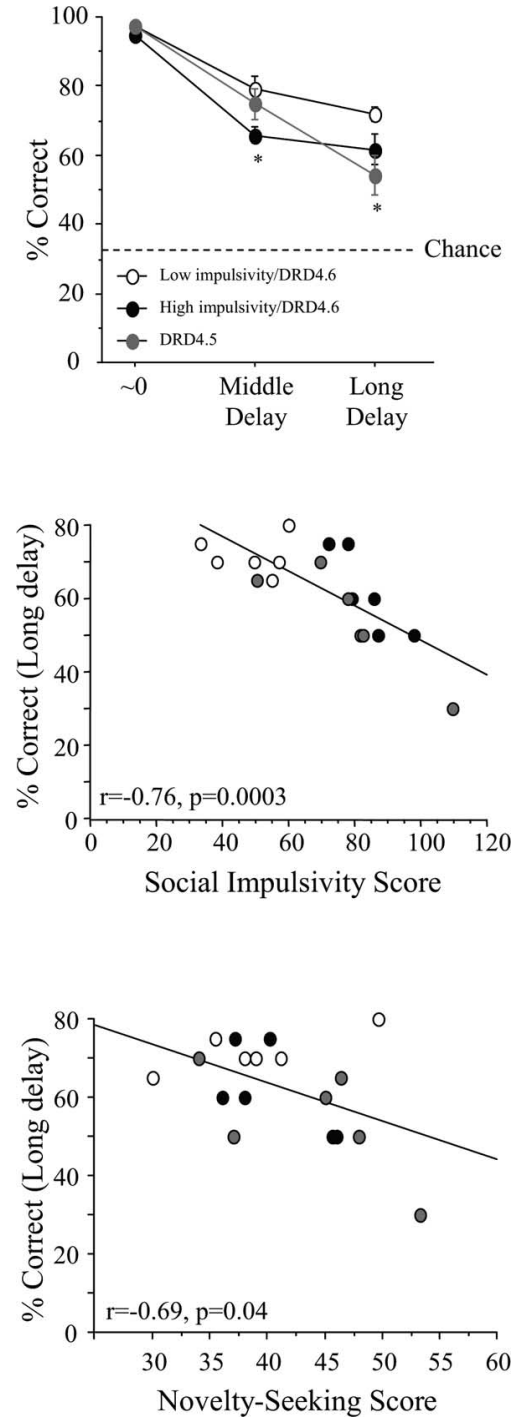

Figure 2. Delay-accuracy curves for low-impulsive/DRD4.6, high-impulsive/DRD4.6 and DRD4.5 subjects (top panel). Both the high-impulsivity/DRD4.6 and DRD4.5 groups exhibited delay-dependent deficits on the spatial delayed response task when compared with the lowimpulsivity/DRD4.6 group. Choice accuracy at the longest delays correlates inversely with Social Impulsivity scores (middle panel) and the Novelty Seeking score (bottom panel). Data points represent mean \pm SEM (top panel) or data points for individual subjects (middle and bottom panels). ${ }^{*} p<0.05$ : significantly different from the low-impulsivity/DRD4.6 group.

nificant $(r=-0.76 ; p=0.003)$, with impulsivity negatively relating to working memory performance, as expected. To be sure that this relationship was not being driven solely by group differences in the high-and low-impulsivity groups, data from only the 12 monkeys constituting the high-impulsivity/DRD4.6 and DRD4.5 groups were examined for the relationship between social impulsivity and working memory performance. Again, choice accuracy under long delays was negatively correlated with Social Impulsivity scores (Fig. 2$)(r=-0.75 ; p=0.005)$. There- 
fore, under conditions in which variation in accuracy is greatest, a significant, negative relationship between working memory performance and impulsivity is present.

The Novelty Seeking scores for the entire cohort were also regressed against correct responding at the medium and long delays. As with the Social Impulsivity measure, the Novelty Seeking score was inversely related to working memory performance at long delays (Fig. 2) $(r=-0.69 ; p=0.04)$ but not medium delays $(r=-0.15 ; p=0.9)$ (data not shown).

A multiple regression model was used to determine whether the Social Impulsivity and Novelty Seeking indices explained the same or nonoverlapping proportions of the variance in choice accuracy on long delay trials. When the Novelty Seeking score was added to the regression model after the Social Impulsivity measure, the combined model explained more variance $(r=$ $-0.86 ; p=0.002)$ than did the Social Impulsivity score alone $(r=$ $-0.75 ; p=0.005)$. Moreover, when controlling for the Social Impulsivity score, the Novelty Seeking Index was still significantly inversely correlated with working memory performance for long delays $(r=-0.46 ; p=0.04)$.

\section{CSF monoamine metabolite analyses}

In this relatively small cohort of subjects, CSF levels of 5-HIAA and HVA were not significantly different among the groups (Fig. 3) $\left(\mathrm{F}_{(2,14)}=0.88, p=0.43\right.$, and $\mathrm{F}_{(2,14)}=0.56, p=0.59$, respectively). Nevertheless, an interesting trend appeared when metabolite levels were plotted against working memory performance at the longest delays. The correlation between 5-HIAA levels and choice accuracy just failed to reach significance (Fig. 3) $(r=$ $-0.47 ; p=0.059)$; the trend was inverse, such that monkeys with the highest 5-HIAA levels performed the most poorly when delays were long. If the monkeys in the high-impulsivity/DRD4.6 and DRD4.5 groups were examined separate from the lowimpulsivity group, the relationship between 5-HIAA and impulsivity reached significance (Fig. 3$)(r=-0.63 ; p=0.03)$. Notably, this relationship seemed to be driven almost entirely by three monkeys in the DRD4.5 group that exhibited high 5-HIAA levels and relatively poor working memory performance. In contrast, the relationship between HVA levels and choice accuracy was not significant for either the entire cohort $(r=-0.25 ; p=0.34)$ (data not shown) or for the high-impulsivity/DRD4.6 and DRD4.5 groups $(r=-0.33 ; p=0.32)$ (data not shown).

\section{Discussion}

The results of these analyses indicate that naturalistic measures of social impulsivity and novelty seeking are predictive of individual differences in spatial working memory performance in the vervet monkey. Highly impulsive animals exhibited poorer response accuracy than did low-impulsivity animals, and correct responding in the delayed response task inversely related to Social Impulsivity and Novelty Seeking scores. Of particular interest, these two factors, while being modestly correlated with one another, predicted at least somewhat different proportions of the variance in working memory performance, meaning that dimensions of impulsivity may impact on working memory performance in qualitatively similar ways but through different mechanisms. These data also suggest that the behavioral impulsivity predicts working memory performance through polygenetic mechanisms.

The relationships between impulsivity and working memory performance were present in animals that carried no or 1 allele of a 5-repeat version of the DRD4 gene previously associated with high novelty seeking (Bailey et al., 2007). Although variation in
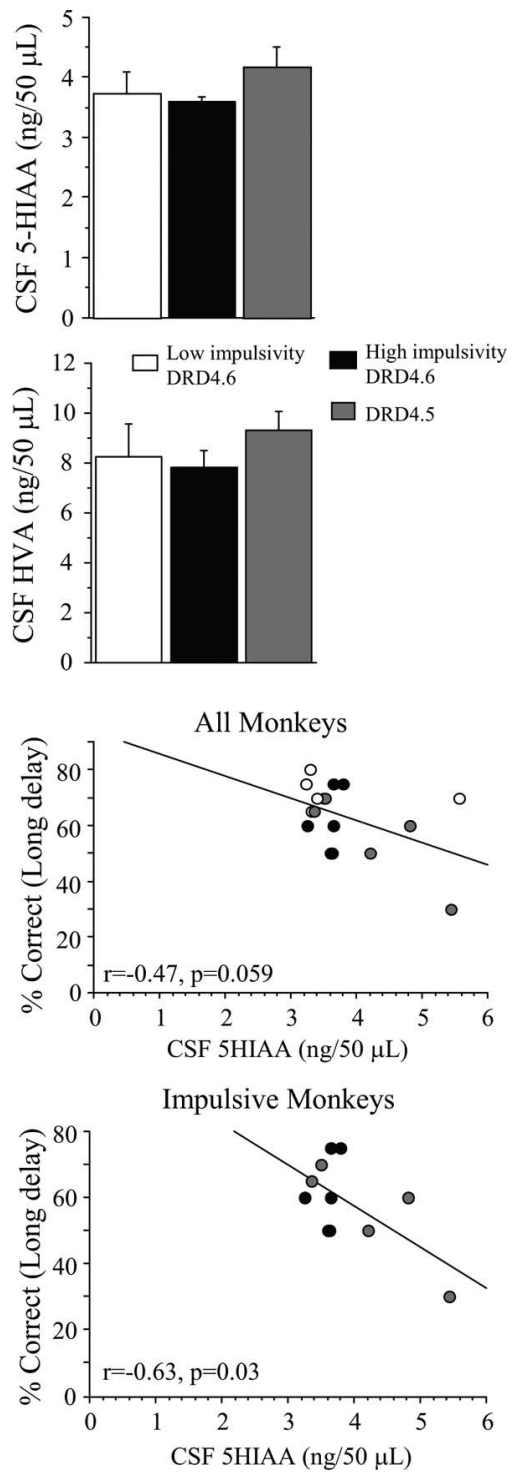

Figure 3. CSF concentrations of the serotonin catabolite 5-HIAA or dopamine catabolite HVA in the three groups of subjects included in this study; no group differences were observed. Working memory maintenance inversely correlates with CSF 5-HIAA concentrations in the highimpulsive/DRD4.6 and DRD4.5 monkeys but the relationship just fails to reach significance if all monkeys are considered. Data represent mean \pm SEM (top panel) or data points for individual subjects (middle and bottom panels).

the DRD4 gene did not predict significant differences in novelty seeking in this small cohort, our data does indicate that the genetic locus influences complex aspects of temperament and associated variation in cognition. Nevertheless, our results also indicate that other genetic mechanisms associate with phenotypic variation in these domains in a qualitatively similar manner. Together with a recently reported association between impulsivity and working memory-related brain activation, assessed using fMRI, in young adult humans (Cools et al., 2007), this study suggests that ADHD-related behavioral and cognitive phenotypes are closely linked, possibly through a common set of genetic and neurochemical determinants.

\section{Heritability and genetic factors}

The behavioral responses indicative of impulsivity that were used to select monkeys into this study, approach and aggression during exposure to a potentially threatening stranger, are heritable 
traits that appear to depend on partially overlapping genetic and neurochemical mechanisms (Fairbanks, 2001; Fairbanks et al., 2001, 2004). Here, we show that approach to a nonsocial novel stimulus (novelty seeking) modestly correlates with the impulsivity score derived in the intruder challenge test. Also, monkeys selected into the study according to the 5-repeat version of the DRD4 gene, previously shown to associate with high novelty seeking (Bailey et al., 2007), exhibit greater social impulsivity. The current data support the conclusion that these measures index a common, ecologically valid construct with potential relevance to ADHD symptoms; however, they also interrogate nonoverlapping processes, as evidenced by their somewhat independent associations with working memory performance.

Recent studies have indicated that maintenance and manipulation subcomponents of spatial working memory demonstrate substantial heritability in humans (Ando et al., 2001; TuulioHenriksson et al., 2002; Glahn et al., 2003; Gur et al., 2007). Similar studies have yet to be performed in pedigreed monkeys or other nonhuman animals, but the relationship between heritable aspects of impulsivity and working memory in humans (Cools et al., 2007) and monkeys (current data) raises the possibility that this cognitive domain could share genetic variance with the temperamental trait.

\section{Monoaminergic mechanisms}

Previous studies in macaques and vervet monkeys have highlighted the role for CNS serotonergic function in social impulsivity (Higley et al., 1996; Westergaard et al., 1999; Fairbanks et al., 2001; Manuck et al., 2003; Westergaard et al., 2003); in both species, low CSF concentrations of the serotonin catabolite 5-HIAA are associated with greater behavioral impulsivity. In this small cohort, 5-HIAA levels were not different between high- and low-impulsive subjects, nor did 5-HIAA levels correlate with behavioral impulsivity. However, CSF 5-HIAA concentrations in impulsive animals were inversely related to working memory, an effect opposite to that predicted. Importantly, this correlation appeared to be driven by relatively high monoamine turnover in animals carrying the DRD4.5 allele. It is possible, therefore, that the association between 5-HIAA and working memory is related only to independent effects of DRD4 genotype on cognition and monoamine turnover. Future research should examine whether genetic variation in the DRD4 gene associates with specific adaptations in monoamine transmission, particularly in light of previous demonstrations that pharmacological inhibition of the dopamine $\mathrm{D}_{4}$ receptor can lead to increased dopamine turnover under some conditions (Jentsch et al., 1999).

\section{Neural mechanisms: relevance to ADHD}

Collectively, our data indicate that impulsive behavior is a strong indicator of individual differences in working memory maintenance, possibly because of shared neural mechanisms that modulate both phenotypes. Specific subregions of the frontal cortex play important, although not exclusive, roles in the cognitive control over behavior, facilitating the execution of planned actions rather than simple emission of stimulus-bound behavior (Dias et al., 1996; Goldman-Rakic, 1996; Miller and Cohen, 2001; Chamberlain et al., 2006). Although the mechanisms of working memory and inhibitory control over behavior appear to be somewhat independent (Roberts et al., 1994; Miller and Cohen, 2001; Garavan et al., 2002; Dalley et al., 2004), they are also likely to be interactive when complex, planned responses require the suppression of rapid, prepotent actions. For these reasons, cellular or circuitry differences within dorsal and ventral aspects of the lat- eral frontal cortex could hypothetically differentiate impulsive and nonimpulsive animals, and these biological substrates could directly contribute to poor working memory performance and, consequently, to reduced capacity to inhibit inappropriate behavior.

Whether other core symptoms of ADHD (hyperactivity or inattentiveness) or other endophenotypes (poor response inhibition) also associate with naturalistic measures of impulsivity remain to be examined, but the association reported here supports the use of the impulsive monkey to investigate genetic, genomic and neurochemical mechanisms that drive symptomatic aspects of ADHD-like traits. The reported relationship between DRD4 and behavior in both man and monkey already underscores the degree to which nonhuman primates can illuminate basic mechanisms controlling complex human phenotypes. Future studies of these temperamental and cognitive traits should generate unexpected insights into the genetic and genomic structures relevant to impulsivity-related phenotypes.

\section{References}

Adams BW, Bradberry CW, Moghaddam B (2002) NMDA antagonist effects on striatal dopamine release: microdialysis studies in awake monkeys. Synapse 43:12-18.

Ando J, Ono Y, Wright MJ (2001) Genetic structure of spatial and verbal working memory. Behav Genet 31:615-624.

Arnsten AF (2006) Stimulants: therapeutic actions in ADHD. Neuropsychopharmacology 31:2376-2383.

Aron AR, Poldrack RA (2005) The cognitive neuroscience of response inhibition: relevance for genetic research in attention-deficit/hyperactivity disorder. Biol Psychiatry 57:1285-1292.

Bailey JN, Breidenthal SE, Jorgensen MJ, McCracken JT, Fairbanks LA (2007) The association of DRD4 and novelty seeking is found in a nonhuman primate model. Psychiatr Genet 17:23-27.

Blondeau C, Dellu-Hagedorn F (2007) Dimensional analysis of ADHD subtypes in rats. Biol Psychiatry 61:1340-1350.

Castellanos FX, Tannock R (2002) Neuroscience of attention-deficit/hyperactivity disorder. Nat Rev Neurosci 3:617-628.

Chamberlain SR, Muller U, Robbins TW, Sahakian BJ (2006) Neuropharmacological modulation of cognition. Curr Opin Neurol 19:607-612.

Chamberlain SR, Robbins TW, Sahakian BJ (2007) The neurobiology of attention-deficit/hyperactivity disorder. Biol Psychiatry 61:1317-1319.

Clark L, Blackwell AD, Aron AR, Turner DC, Dowson J, Robbins TW, Sahakian BJ (2007) Association between response inhibition and working memory in adult ADHD: a link to right frontal cortex pathology? Biol Psychiatry 61:1395-1401.

Cools R, Sheridan M, Jacobs E, D’Esposito M (2007) Impulsive personality predicts dopamine-dependent changes in frontostriatal activity during component processes of working memory. J Neurosci 27:5506-5514.

Dalley JW, Cardinal RN, Robbins TW (2004) Prefrontal executive and cognitive functions in rodents. Neurosci Biobehav Rev 28:771-784.

Davids E, Zhang K, Tarazi FI, Baldessarini RJ (2003) Animal models of attention-deficit hyperactivity disorder. Brain Res Brain Res Rev 42:1-21.

Dellu-Hagedorn F (2006) Relationship between impulsivity, hyperactivity and working memory: a differential analysis in the rat. Behav Brain Funct $2: 10$.

Dias R, Robbins TW, Roberts AC (1996) Dissociation in prefrontal cortex of affective and attentional shifts. Nature 380:69-72.

Dowson JH, Blackwell AD, Turner DC, Harvey E, Malhotra T, Robbins TW, Sahakian BJ (2007) Questionnaire ratings of attention-deficit/hyperactivity disorder (ADHD) in adults are associated with spatial working memory. Eur Psychiatry 22:256-263.

Fairbanks LA (2001) Individual differences in response to a stranger: social impulsivity as a dimension of temperament in vervet monkeys (Cercopithecus aethiops sabaeus). J Comp Psychol 115:22-28.

Fairbanks LA, Melega WP, Jorgensen MJ, Kaplan JR, McGuire MT (2001) Social impulsivity inversely associated with CSF 5-HIAA and fluoxetine exposure in vervet monkeys. Neuropsychopharmacology 24:370-378.

Fairbanks LA, Newman TK, Bailey JN, Jorgensen MJ, Breidenthal SE, Ophoff RA, Comuzzie AG, Martin LJ, Rogers J (2004) Genetic contributions to 
social impulsivity and aggressiveness in vervet monkeys. Biol Psychiatry 55:642-647.

Faraone SV, Perlis RH, Doyle AE, Smoller JW, Goralnick JJ, Holmgren MA, Sklar P (2005) Molecular genetics of attention-deficit/hyperactivity disorder. Biol Psychiatry 57:1313-1323.

Garavan H, Ross TJ, Murphy K, Roche RAP, Stein EA (2002) Dissociable executive functions in the dynamic control of behavior. NeuroImage 17:1820-1829.

Glahn DC, Therman S, Manninen M, Huttunen M, Kaprio J, Lonnqvist J, Cannon TD (2003) Spatial working memory as an endophenotype for schizophrenia. Biol Psychiatry 53:624-626.

Goldman-Rakic PS (1987) Circuitry of the primate prefrontal cortex and the regulation of behavior by representational memory. In: Handbook of physiology, the nervious system, higher functions of the brain (Plum F, ed), pp 373-417. Bethesda, MD: American Psychological Society.

Goldman-Rakic PS (1996) The prefrontal landscape: implications of functional architecture for understanding human mentation and the central executive. Philos Trans R Soc Lond B Biol Sci 351:1445-1453.

Gur RE, Calkins ME, Gur RC, Horan WP, Nuechterlein KH, Seidman LJ, Stone WS (2007) The Consortium on the Genetics of Schizophrenia: neurocognitive endophenotypes. Schizophr Bull 33:49-68.

Higley JD, Mehlman PT, Poland RE, Taub DM, Vickers J, Suomi SJ, Linnoila M (1996) CSF testosterone and 5-HIAA correlate with different types of aggressive behaviors. Biol Psychiatry 40:1067-1082.

Jentsch JD, Taylor JR, Redmond Jr DE, Elsworth JD, Youngren KD, Roth RH (1999) Dopamine D4 receptor antagonist reversal of subchronic phencyclidine-induced object retrieval/detour deficits in monkeys. Psychopharmacology (Berl) 142:78-84.

Keltikangas-Jarvinen L, Elovainio M, Kivimaki M, Lichtermann D, Ekelund J, Peltonen L (2003) Association between the type 4 dopamine receptor gene polymorphism and novelty seeking. Psychosom Med 65:471-476.

Lichter JB, Barr CL, Kennedy JL, Van Tol HH, Kidd KK, Livak KJ (1993) A hypervariable segment in the human dopamine receptor D4 (DRD4) gene. Hum Mol Genet 2:767-773.

Lusher JM, Chandler C, Ball D (2001) Dopamine D4 receptor gene (DRD4) is associated with Novelty Seeking (NS) and substance abuse: the saga continues. Mol Psychiatry 6:497-499.

Manuck SB, Kaplan JR, Rymeski BA, Fairbanks LA, Wilson ME (2003) Approach to a social stranger is associated with low central nervous system serotonergic responsivity in female cynomolgus monkeys (Macaca fascicularis). Am J Primatol 61:187-194.

Miller EK, Cohen JD (2001) An integrative theory of prefrontal cortex function. Annu Rev Neurosci 24:167-202.

Paterson AD, Sunohara GA, Kennedy JL (1999) Dopamine D4 receptor gene: novelty or nonsense? Neuropsychopharmacology 21:3-16.

Roberts AC, De Salvia MA, Wilkinson LS, Collins P, Muir JL, Everitt BJ, Robbins TW (1994) 6-Hydroxydopamine lesions of the prefrontal cortex in monkeys enhance performance on an analog of the Wisconsin Card Sort Test: possible interactions with subcortical dopamine. J Neurosci 14:2531-2544.

Russell VA, Sagvolden T, Johansen EB (2005) Animal models of attentiondeficit hyperactivity disorder. Behav Brain Funct 1:9.

Sagvolden T, Russell VA, Aase H, Johansen EB, Farshbaf M (2005) Rodent models of attention-deficit/hyperactivity disorder. Biol Psychiatry 57:1239-1247.

Smalley SL, Bailey JN, Palmer CG, Cantwell DP, McGough JJ, Del'Homme MA, Asarnow JR, Woodward JA, Ramsey C, Nelson SF (1998) Evidence that the dopamine D4 receptor is a susceptibility gene in attention deficit hyperactivity disorder. Mol Psychiatry 3:427-430.

Solanto MV (2001) Attention-deficit/hyperactivity disorder: clinical features. In: Stimulant drugs and ADHD: basic and clinical neuroscience (Solanto MV, Arnsten AF, Castellanos FX, eds), pp 3-30. New York: Oxford UP.

Swanson JM, Flodman P, Kennedy J, Spence MA, Moyzis R, Schuck S, Murias M, Moriarity J, Barr C, Smith M, Posner M (2000) Dopamine genes and ADHD. Neurosci Biobehav Rev 24:21-25.

Tuulio-Henriksson A, Haukka J, Partonen T, Varilo T, Paunio T, Ekelund J, Cannon TD, Meyer JM, Lonnqvist J (2002) Heritability and number of quantitative trait loci of neurocognitive functions in families with schizophrenia. Am J Med Genet 114:483-490.

Westergaard GC, Suomi SJ, Higley JD, Mehlman PT (1999) CSF 5-HIAA and aggression in female macaque monkeys: species and interindividual differences. Psychopharmacology (Berl) 146:440-446.

Westergaard GC, Suomi SJ, Chavanne TJ, Houser L, Hurley A, Cleveland A, Snoy PJ, Higley JD (2003) Physiological correlates of aggression and impulsivity in free-ranging female primates. Neuropsychopharmacology 28 : 1045-1055. 